\title{
A Comparative Study of Different Texture Segmentation Techniques
}

\author{
Madasu Hanmandlu ${ }^{1}$, Shilpa Agarwal ${ }^{2}$, and Anirban Das ${ }^{3}$ \\ ${ }^{1}$ Dept. of Electrical Engineering, I.I.T Delhi, Hauz Khas, \\ New Delhi 110 016, India \\ mhmandludee.iitd.ernet. in \\ ${ }^{2}$ Dept. of Electrical Engineering,I.I.T Delhi, Hauz Khas, \\ New Delhi 110 016, India \\ shilpadce@yahoo.com \\ ${ }^{3}$ Dept. of Computer Science, Jamia Millia Islamia University, \\ New Delhi 110 025, India \\ anirban_das_jnu@rediffmail.com
}

\begin{abstract}
Image segmentation techniques are very useful in the analysis of aerial images, biomedical images and seismic images as well as in the automation of industrial applications. The paper presents a few texture-specific features for the segmentation of texture images. We will present four approaches to texture segmentation, viz, descriptor based, heuristic function based, defuzzified feature based and Mask feature based. We have mainly used FCM for texture segmentation. Keywords- Texture segmentation, entropy, descriptors, texture energy measure, defuzzified feature
\end{abstract}

\section{Introduction}

The segmentation of textures requires the choice of proper texture-specific features with good discriminative power [1]. Generally speaking, texture feature extraction methods can be classified into three major categories, namely, statistical, structural and spectral. Various texture descriptors have been proposed in the literature. In addition to the aforementioned methods, Law's texture energy measures, Markov random field models, texture spectrum etc., are some other texture descriptors. A texture can be either regular pattern like man made texture, for e.g., "Mat" or "Fence" or it can be a random texture as in Natural images. We have implemented several methods for segmentation of Natural as well as mosaic images.

The paper is organized as follows: Section 2 gives an overview of the descriptor based approach for texture segmentation. In section 3, the feature based on heuristic function is explained. In Section 4, an overview of the mask-based feature for texture segmentation is discussed. Section 5 presents a comparison of results from different texture segmentation techniques.

\section{Texture Image Segmentation Using Descriptors}

The ambiguities in texture that arise due to fuzzy nature of image function give an opportunity to devise fuzzy texture features [2]. Since texture is region based, we consider arrangement of image functions (i.e., intensities) of pixels in a local region, 
say, a window, in order to characterize the texture. Spatial interaction model is used in the present work to evolve texture features. This approach brings into consideration the spatial arrangement of pixel intensities in a window. A pixel having similar intensity as that of its neighborhood will have a higher correlation.

The correlation of pixel $x(j)$ about its neighborhood $x(i)$ in a window of size pxp can be represented by a Gaussian membership function, which depends on the difference in their gray values. The membership function is defined as

$$
\mu_{j}(i)=\exp \left[-\left\{\frac{x(j)-x(i)}{\tau}\right\}^{2}\right] \mathrm{j}=1,2, \ldots \ldots \mathrm{n}
$$

where $\tau$ is the fuzzifier selected as $10 \%$ of the size of window. We have calculated the membership value with the maximum gray level in a window as the reference. Accordingly, the membership function in (1) is modified to:

$$
\mu_{j}(i)=\exp \left[-\left\{\frac{x(j)-x_{\max }}{\tau}\right\}^{2}\right] \mathrm{j}=1,2, \ldots \ldots 8
$$

The membership value is $\mu_{j}(i)=1.0$ for $x(j)=x_{\max }$. The membership values are less than or equal to 1 for the neighboring pixels in the window. Next, we obtain the cumulative response to the current pixel, $\mathrm{x}$ by taking weighted sum. So we have

$$
y_{i}(x)=\sum_{j=1}^{n} \frac{\mu_{j}(i) \cdot x(j)}{\sum_{j=1}^{n} \mu_{j}(i)}
$$

Where $\mathrm{n}$ is the number of pixels in a window. The output $\mathrm{y}(\mathrm{x})$ will be maximum when all the neighboring pixels are of the same value. Following this, the cumulative responses of all pixels are computed by forming $3 \times 3$ windows on original $512 \times 512$ image giving rise to an output image.

\subsection{Texture Descriptors}

If we use all the elements of $Y\left(Y=\left\{y_{i}(x)\right\}\right)$, we would be bogged down by the computational problem. Instead, we derive texture descriptors that capture the essence of the texture subimage. If $Y$ is of size $M x M$, it is then partitioned into sub-images, each of size $w \times w$ where $w<<M$. We compute the n-descriptors for each sub-images, which results into a descriptor matrix, $D$ of size $R \times R$, where $R=M / w$. The element of $D$ is a vector $\left[d_{1}, d_{2}, \ldots d_{n}\right]^{\mathrm{T}}$, whose size $n$ is equal to the number of descriptors. We want to capture the underlying structure of a texture through a few descriptors Maximum occurrence, Difference moment of order $k($ for $\mathrm{k}=2)$ (also termed as 'inertia' feature), Entropy, Measures the similarity (also called 'Energy' feature).

\section{Heuristic Function Based Feature}

The following heuristic function is used to calculate the texture feature. This has been devised by experimentation.

$$
E_{T}=\sum p(x)\left[t^{2} f(x)+t a f(x)+b\right]
$$


The parameter " $\mathrm{t}$ " is obtained by optimizing the expression for $\mathrm{E}_{\mathrm{T}}$.

$$
\therefore t=\frac{-a \sum f(x)}{\sum p(x) f(x)}
$$

Here $p(x)$ is the defuzzified response of the current pixel and $f(x)$ is the fractal dimension for the current window. The expression for $\mathrm{p}(\mathrm{x})$ and $\mathrm{f}(\mathrm{x})$ are derived as under.

$$
p(x)=\sum_{j=1}^{n} \frac{\mu_{j}(i) \cdot x(j)}{\sum_{j=1}^{n} \mu_{j}(i)} \quad \cdots . .(6) \quad \text { with } \mu_{j}(i)=\exp \left[-\left\{\frac{x(j)-x_{\max }}{\tau}\right\}^{2}\right] \mathrm{j}=1,2 \ldots 8
$$

Where $x_{\max }$ is the max gray level in current window with respect to which the gray levels of all neighboring pixels $x(j)$ are compared and $\tau$ is the fuzzifier which is taken to be the initial size of the window.

Let us take $(\chi)$ as the initial window size for calculating the defuzzified response. Then fractal dimension is defined as

$$
f_{i}(x)=\sum_{j=1}^{n} \ln _{s_{j}}\left\{\frac{\mathrm{y}_{\mathrm{i}}(\mathrm{s} \chi)}{\mathrm{y}_{\mathrm{i}}(\chi)}\right\} .
$$

\section{Texture Feature Based on Masks for Image Enhancement}

Laws' Texture Energy Measures (TEM) [3] determine textural properties by assessing average gray-level, edges, spots, ripples, and waves in texture. The measures are derived from three simple vectors: $\mathrm{L} 3=(1,2,1)$, E3 $=(-1,0,1), \mathrm{S} 3=(-1,2,-1)$. After convolution of these vectors with themselves and each other, five vectors will result: L5 $=1,4,6,4,1)$, E5 = $(-1,-2,0,2,1)$, S5 $=(-1,0,2,0,-1)$, R5 $=(1,-4,6,-4,1)$, W5 $=(-1,2,0$, $-2,-1)$ where L5 represents local averaging. S5 and E5 are spot and edge detectors; R5 can be used as "ripple" detector and W5 as "wave" detector.

The texture value of the pixel at coordinate $(\mathrm{i}, \mathrm{j})$ is defined as

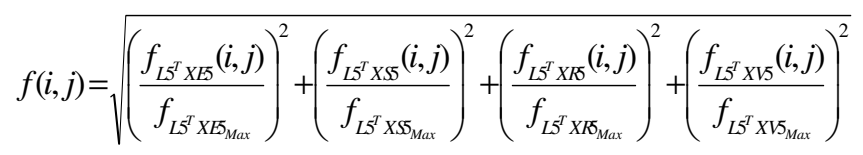

Where $f_{L 5^{T} X E 5}(i, j), f_{L 5^{T} X S 5}(i, j), f_{L 5^{T} X R 5}(i, j)$ and $f_{L 5^{T} X W 5}(i, j)$ are the convoluted results of the image with the four masks. These texture features can be directly used for segmentation.

\section{Results of Comparison of Different Texture Features for Texture Segmentation}

This section gives the simulation results for the Fuzzy Descriptors based texture segmentation. The descriptors used are Entropy, Energy, Inertia and Frequency. The segmentation is done using Fuzzy C-Means for both the Natural as well as mosaic images. 


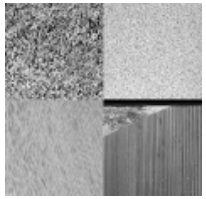

Original Mosaic-1

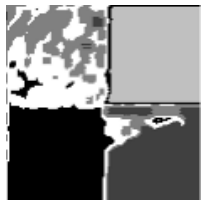

Segmented Mosaic-1

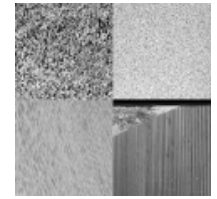

Original Mosaic-1

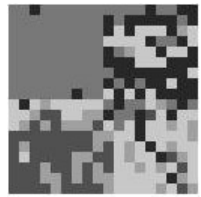

Segmented Mosaic-1

Fig. 1. Results for Mosaic Image-1 for segmentation using Descriptors

Fig. 2. Results for Mosaic Image-1 for segmentation using Heuristic Function

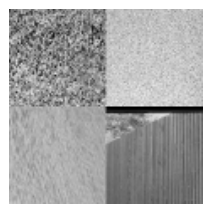

Original Mosaic-1

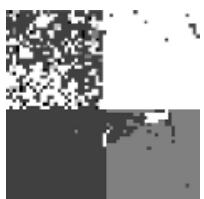

Segmented Mosaic-1

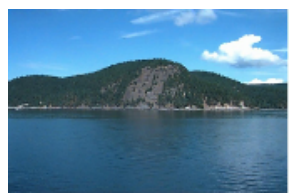

Original Natural-1

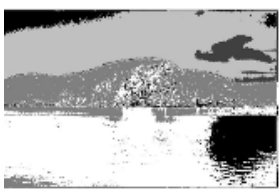

Segmented Natural-1

Fig. 3. Results for Natural Image-1 for segmentation using Defuzzified Feature

Fig. 4. Results for Natural Image-1 for segmentation using Mask based Feature

\section{Summary and Conclusions}

In this paper, we have experimented on several features for texture segmentation keeping the clustering technique the same. The performance of descriptor-based approach is dependent on the surrounding texels. Heuristic based approach is found to be useful for random textures because of its property to capture randomness. The defuzzified feature based approach is amenable for regular patterns/textures. The mask- based approach is immensely appropriate for natural textures.

\section{References}

1. Chaudhuri, B.B., Sarkar, N.: Texture Segmentation Using Fractal Dimension. IEEE Transactions on Pattern Analysis and Machine Intelligence, Vol 17,No.1, 1995

2. Hanmandlu, M., Madasu, V. K., Vasikalra, S.: A Fuzzy Approach to Texture Segmentation. Proceeding of ITCC'04: Coding and Computing, 2004.

3. Laws, K.I.: Texture Energy Measures. DARPA Image Understanding Workshop. Los Angeles, CA, pages 47-51, DARPA, Los Altos, CA, 1979. 